\title{
ON A PROBLEM OF S. BANACH FROM THE SCOTTISH BOOK
}

\author{
K. S. KAZARIAN
}

(Communicated by J. Marshall Ash)

\begin{abstract}
Denote by $B(\Phi)$ the closure in $L^{2}$ of linear combinations of functions of the orthonormal system $\Phi=\left\{\Phi_{n}\right\}_{n=1}^{\infty}$. Denote by $B(\Phi)^{\perp}$ the orthogonal complement of $B(\Phi)$ in $L^{2}$. We prove the following theorem: Let $\varphi$ be a nonnegative measurable function defined on $[0,+\infty)$ such that $\lim _{x \rightarrow+\infty} x^{2} / \varphi(x)=0$ and $N$ is a positive integer or $N=+\infty$. Then there is a uniformly bounded orthonormal system $\Phi^{(N)}=\left\{\Phi_{n}\right\}_{n=1}^{\infty}$ such that $\operatorname{dim} B\left(\Phi^{(N)}\right)^{\perp}=N$ and, for every nontrivial function $f$ from $B\left(\Phi^{(N)}\right)^{\perp}$, $\int \varphi(|f(t)|) d t=+\infty$.
\end{abstract}

In the present paper we prove a theorem which, in particular, gives an answer to the following problem of S. Banach [1, p. 161].

Given a sequence of functions $\Phi=\left\{\Phi_{n}(t)\right\}_{n=1}^{\infty}$ orthogonal, normed, measurable and uniformly bounded, i.e.,

$$
\left|\Phi_{n}(t)\right| \leq M, \quad t \in[a, \mathscr{C}], n=1,2, \ldots,
$$

can one always complete it, using functions with the same bound, to a sequence which is orthogonal, normed and complete? Consider the case when infinitely many functions are necessary for completion.

Denote by $B(\Phi)$ the closure in $L^{2}$ of linear combinations of functions of the system $\Phi$. Denote by $B(\Phi)^{\perp}$ the orthogonal completion in $L^{2}$ of the subspace $B(\Phi)$. In the formulated problem the last condition means that $\operatorname{dim} B(\Phi)^{\perp}=+\infty$. Probably, for the case $\operatorname{dim} B(\Phi)^{\perp}<+\infty$, S. Banach knew how to construct a counterexample for his problem. Let $\varphi$ be a nonnegative measurable function defined on $[0,+\infty)$, the symbol $\varphi(L)$ will denote the class of all the measurable functions such that $\int_{0}^{1} \varphi(|f(t)|) d t<+\infty$.

Theorem. Suppose that $\varphi$ is a nonnegative measurable function defined on $[0,+\infty)$ such that

$$
\lim _{x \rightarrow+\infty} \frac{x^{2}}{\varphi(x)}=0
$$

Received by the editors August 15, 1988 and, in revised form, February 14, 1989. 1980 Mathematics Subject Classification (1985 Revision). Primary 42C05; Secondary 42C30. Key words and phrases. Uniformly bounded orthonormal system, orthogonal completion. 
and $N$ is a positive integer or $N=+\infty$. Then there exists an orthonormal system $(O N S) \Phi^{(N)}=\left\{\Phi_{n}\right\}_{n=1}^{\infty}$ such that (1) is satisfied, $\operatorname{dim} B\left(\Phi^{(N)}\right)^{\perp}=N$ and

$$
B\left(\Phi^{(N)}\right)^{\perp} \cap \varphi(L)=\{0\},
$$

where $\{0\}$ denotes the trivial element of the space $L^{2}$.

When this article was prepared for publication, B. S. Kašin kindly sent me some materials which informed me that, in 1985 , he and A. M. Olevskii wrote an article where, with the help of probabilistic methods, they proved a similar theorem for $N=+\infty$ with

$$
B\left(\Phi^{(\infty)}\right)^{\perp} \cap L^{\infty}=\{0\}
$$

instead of (3). B. S. Kašin also communicated that, in $\left(3^{\prime}\right)$, they cannot change $L^{\infty}$ by some $L^{p}, p>2$. Also, from a letter of Z. Ciesielski to B. Kašin, I learned that "this problem in 1936 was solved by S. Kaczmarz [2] with the help of S. Banach method." Unfortunately that is all that I know about the article of S. Kaczmarz.

We will first present an easy proof of the fact that there is an ONS $\Phi^{(\infty)}=$ $\left\{\Phi_{n}\right\}$, such that $\operatorname{dim} B\left(\Phi^{(\infty)}\right)^{\perp}=+\infty$ and the subspace $B\left(\Phi^{(\infty)}\right)^{\perp}$ has no uniformly bounded orthonormal basis. In our constructions, the Haar system and the special matrices $H_{k}$ (see $\left.[3, \S 7.9 ; 4]\right)$ originated from the Haar system will be used. Recall the definition of the Haar system

$$
\chi_{k}^{(j)}(x)= \begin{cases}2^{k / 2}, & \text { if }(2 j-2) / 2^{k+1}<x<(2 j-1) / 2^{k+1}, \\ -2^{k / 2} & \text { if }(2 j-1) / 2^{k+1}<x<2 j / 2^{k+1}, \\ 0, & \text { if } x \notin\left[(j-1) / 2^{k}, j / 2^{k}\right],\end{cases}
$$

where $k=0,1, \ldots, 1 \leq j \leq 2^{k}$. The value at an interior discontinuity point is to be equal to the arithmetic mean of the one-sided limits at this point, and the value at an endpoint is to be just the appropriate one-sided limit value. The Haar system in its ordinary numeration is defined as follows:

$$
\chi_{0}^{(0)}(x)=\chi_{1}(x)
$$

and

$$
\chi_{k}^{(j)}(x)=\chi_{n}(x), \quad \text { for } n=2^{k}+j, \quad k=0,1, \ldots, 1 \leq j \leq 2^{k} .
$$

To define the special matrices $H_{k}$ arising from the Haar system, for any positive integer $k$, take the midpoints $x_{\zeta}^{(k)}=(\zeta+1 / 2) 2^{-k}$ of the intervals $\left(\zeta \cdot 2^{-k}\right.$, $\left.(\zeta+1) 2^{-k}\right)$ and let

$$
H_{k}=\left\{h_{i j}^{(k)}=2^{-k / 2} \chi_{i}\left(x_{j}^{(k)}\right): 1 \leq i \leq 2^{k}, 0 \leq j \leq 2^{k}-1\right\} .
$$

We now prove the following weak version of our theorem: There exists uniformly bounded ONS $\Phi^{(\infty)}=\left\{\Phi_{n}\right\}_{n=1}^{\infty}$ such that $\operatorname{dim} B\left(\Phi^{(\infty)}\right)^{\perp}=+\infty$ and the subspace $B\left(\Phi^{(\infty)}\right)^{\perp}$ has no uniformly bounded orthonormal basis. 
Proceed with the following scheme: break the Haar system into two subsystems $X=\left\{\chi_{2 k}^{\left(2^{2 k}-1\right)}(x)\right\}_{k=1}^{\infty}$ and $X^{\prime}=\left\{\chi_{n}\right\}_{n=1}^{\infty} \backslash X$. Then construct a uniformly bounded ON basis $\Phi^{(\infty)}=\left\{\Phi_{n}\right\}_{n=1}^{\infty}$ of the subspace $B\left(X^{\prime}\right)$ and show that it is impossible to complete it in $L_{[0,1]}^{2}$ using uniformly bounded functions. The odd "packets" of the Haar system $X_{1}=\left\{\chi_{2 k+1}^{(j)}(x), 1 \leq j \leq 2^{2 k+1}\right\}_{k=0}^{+\infty}$ are wholly contained in the subsystem $X^{\prime}$. We transform every "packet" in the subsystem $X_{1}$ with the help of the matrices $H_{k}$ as follows:

$$
\psi_{k}^{(i)}(x)=\sum_{j=0}^{2^{2 k}-1} h_{i j}^{(2 k+1)} \chi_{2 k+1}^{(j+1)}(x), \quad k=0,1, \ldots, 1 \leq i \leq 2^{2 k+1} .
$$

By Equations (4)-(7) it obviously follows that $\Psi=\left\{\psi_{k}^{(1)}\right\}_{k=0}^{\infty}$ is a subsystem of the Rademacher system. The collection of the functions $X^{\prime} \backslash X_{1}$ and $\left\{\psi_{k}^{(i)}, 2 \leq i \leq 2^{2 k+1}\right\}_{k=0}^{+\infty}$ are numbered in some order and are denoted by $\left\{g_{j}(x)\right\}_{j=1}^{\infty}$.

Let $\{k(n)\}_{n=1}^{\infty}$ be a sequence of positive integers such that

$$
2^{k(n)-1 / 2} \leq\left\|g_{n}\right\|_{\infty} \leq 2^{k(n) / 2} .
$$

Define an increasing sequence of positive integers $\{\nu(n)\}_{n=1}^{\infty}$ such that

$$
\nu(0)=0, \quad \nu(n)=\nu(n-1)+2^{k(n)}-1 .
$$

The functions of the systems $\left\{g_{j}(x)\right\}_{j=1}^{\infty}$ and $\left\{\psi_{k}^{(1)}(x)\right\}_{k=0}^{\infty}$ will be grouped in "packets", where the $n$th "packet" consists of the function $g_{n}$ and the functions $\left\{\psi_{k}^{(1)}\right\}_{k=\nu(n-1)}^{\nu(n)-1}$. Then apply the orthogonal transformation (6) to these functions as follows:

$$
\begin{aligned}
\Phi_{n}^{(i)}(x)=2^{-k(n) / 2} g_{n}(x)+\sum_{j=1}^{2^{k(n)}-1} h_{i j}^{(k(n))} \psi_{\nu(n-1)+j-1}^{(1)}(x), & \\
& n=1,2, \ldots, 1 \leq i \leq 2^{k(n)} .
\end{aligned}
$$

According to $(6)-(10)$ we get

$$
\left|\Phi_{n}^{(i)}(x)\right| \leq 1+\sum_{j=1}^{\infty} 2^{-j / 2}=c, \quad x \in[0,1], n=1,2, \ldots, 1 \leq i \leq 2^{k(n)} .
$$

Let

$$
\Phi_{m}(x)=\Phi_{n}^{(i)}(x), \quad m=\nu(n-1)+i,
$$

$n=1,2, \ldots, 1 \leq i \leq 2^{k(n)}$. From our construction it obviously follows that the system $\Phi^{(\infty)}=\left\{\Phi_{m}(x)\right\}_{m=1}^{\infty}$ is an orthonormal basis of $B\left(X^{\prime}\right)$. The uniform boundedness of this system follows from (11). Let us show that there is not any uniformly bounded orthonormal basis of $B(X)$. Suppose that $\left\{f_{n}\right\}_{n=1}^{\infty}$ is an orthonormal basis of $B(X)$ and

$$
\left|f_{n}(x)\right| \leq c_{1}, \quad x \in[0,1], n=1,2, \ldots .
$$


Then

$$
\begin{aligned}
& f_{n}(x) \stackrel{L^{2}}{=} \sum_{k=1}^{\infty} a_{k}^{(n)} \chi_{2 k}^{\left(2^{2 k}-1\right)}(x), \\
& a_{n}^{(k)}=\int_{0}^{1} f_{n}(t) \chi_{2 k}^{\left(2^{2 k}-1\right)}(t) d t .
\end{aligned}
$$

According to (4), (12) and (13),

$$
\left|a_{n}^{(k)}\right| \leq c_{1} \cdot 2^{-k}, \quad k, n=1,2, \ldots .
$$

From (13), (14) and the Parsevals equality,

$$
\sum_{k=1}^{\infty}\left[a_{k}^{(n)}\right]^{2}=1, \quad n=1,2, \ldots
$$

And, from our assumption that $\left\{f_{n}\right\}_{n=1}^{\infty}$ is an ON basis of $B(X)$, we have

$$
\chi_{2 k}^{\left(2^{2 k}-1\right)}(x)=\sum_{n=1}^{\infty} a_{k}^{(n)} f_{n}(x)
$$

where the coefficients $\left\{a_{k}^{(n)}\right\}$ are defined by (14).

Parsevals' equality gives

$$
\sum_{n=1}^{\infty}\left[a_{k}^{(n)}\right]^{2}=1, \quad k=1,2, \ldots
$$

Let $n_{1}$ be a positive integer such that

$$
c_{1} \cdot 2^{-n_{1}}<1 / 2
$$

Then, from (17), there is a positive integer $m_{1}$ such that

$$
\sum_{k=1}^{n_{1}}\left[a_{k}^{(n)}\right]^{2}<1 / 2, \quad \text { for } n>m_{1} .
$$

From (18) and (15) we have

$$
\sum_{k=n_{1}+1}^{\infty}\left[a_{k}^{(n)}\right]^{2} \leq c_{1}^{2} \cdot 2^{-2\left(n_{1}+1\right)} \cdot 4 / 3<1 / 12 .
$$

The conditions (19) and (20) contradict (16). Consequently, our assumption is false, i.e., there is not any uniformly bounded ON basis in $B(X)$.

For the proof of the theorem, let us define some matrices. Let $\mathscr{C}=\left\{\mathscr{C}_{j}\right\}_{j=1}^{\infty}$ be a sequence of positive numbers such that

$$
\sum_{j=1}^{\infty} \mathscr{C}_{j}^{2}=1
$$


Then we define the matrix $S(\mathscr{C})$ as follows:

(22)

$$
\begin{gathered}
\zeta_{1 j}=\mathscr{C}_{j}, \quad j=1,2, \ldots \\
\zeta_{i j}=\mathscr{C}_{i} \mathscr{C}_{j} /\left[\left(\sum_{k=1}^{i-1} \mathscr{C}_{k}^{2}\right)\left(\sum_{k=1}^{i} \mathscr{C}_{k}^{2}\right)\right]^{1 / 2}, \quad 1 \leq j \leq i-1, \\
\zeta_{i i}=-\left(\sum_{k=1}^{i-1} \mathscr{C}_{k}^{2}\right)^{1 / 2} /\left(\sum_{k=1}^{i} \mathscr{C}_{k}^{2}\right)^{1 / 2}, \quad \zeta_{i j}=0, \quad j>i, \quad i=2,3, \ldots .
\end{gathered}
$$

This implies

$$
\begin{aligned}
\sum_{j=1}^{\infty} \zeta_{i j} \zeta_{1 j}= & \sum_{j=1}^{i-1} \mathscr{C}_{i} \mathscr{C}_{j}^{2} /\left[\left(\sum_{k=1}^{i-1} \mathscr{C}_{k}^{2}\right)\left(\sum_{k=1}^{i} \mathscr{C}_{k}^{2}\right)\right]^{1 / 2} \\
& -\mathscr{C}_{i}\left(\sum_{k=1}^{i-1} \mathscr{C}_{k}^{2}\right)^{1 / 2} /\left(\sum_{k=1}^{i} \mathscr{C}_{k}^{2}\right)^{1 / 2}=0
\end{aligned}
$$

for $i>1$. Hence, taking into account that, for every $i>1$,

we obviously have

$$
\frac{\zeta_{i 1}}{\zeta_{11}}=\frac{\zeta_{i 2}}{\zeta_{12}}=\cdots=\frac{\zeta_{i i-1}}{\zeta_{1 i-1}},
$$

$$
\sum_{j=1}^{\infty} \zeta_{i j} \cdot \zeta_{i^{\prime} j}=0, \quad i \neq i^{\prime}
$$

Using (22) obtains

$$
\begin{aligned}
\sum_{j=1}^{\infty} \zeta_{i j}^{2}= & \sum_{j=1}^{i-1} \mathscr{C}_{i}^{2} \mathscr{C}_{j}^{2} /\left(\sum_{k=1}^{i-1} \mathscr{C}_{k}^{2}\right)\left(\sum_{k=1}^{i} \mathscr{C}_{k}^{2}\right) \\
& +\sum_{k=1}^{i-1} \mathscr{C}_{k}^{2} / \sum_{k=1}^{i} \mathscr{C}_{k}^{2}=1,
\end{aligned}
$$

and (21), (22), for $j>1$, obtains

$$
\begin{aligned}
\sum_{i=1}^{\infty} \zeta_{i j}^{2}= & \mathscr{C}_{j}^{2}+\sum_{k=1}^{j-1} \mathscr{C}_{k}^{2} / \sum_{k=1}^{j} \mathscr{C}_{k}^{2} \\
& +\sum_{i=j+1}^{\infty} \mathscr{C}_{i}^{2} \mathscr{C}_{j}^{2} /\left(\sum_{k=1}^{i-1} \mathscr{C}_{k}^{2}\right)\left(\sum_{k=1}^{i} \mathscr{C}_{k}^{2}\right) \\
= & 1+\lim _{m \rightarrow+\infty} \mathscr{C}_{j}^{2}\left[1-1 / \sum_{k=1}^{j} \mathscr{C}_{k}^{2}+\sum_{i=j+1}^{m} \mathscr{C}_{i}^{2} /\left(\sum_{k=1}^{i-1} \mathscr{C}_{k}^{2}\right)\left(\sum_{k=1}^{i} \mathscr{C}_{k}^{2}\right)\right] \\
= & 1+\mathscr{C}_{j}^{2} \lim _{m \rightarrow+\infty}\left[1-\left(\sum_{k=1}^{m} \mathscr{C}_{k}^{2}\right)^{-1}\right]=1 .
\end{aligned}
$$


The equality (25) is obvious for $j=1$.

We show the proof for $N<+\infty$. Let

$$
\mathscr{C}_{j}=\frac{\alpha}{j}, \quad j=1,2, \ldots, \quad \alpha^{-2}=\sum_{j=1}^{\infty} 1 / j^{2}
$$

in the definition of the matrix $S(\mathscr{C})$. By (2) choose an increasing subsequence of positive integers $\left\{k_{j}\right\}_{j=1}^{\infty}$ such that

$$
\sum_{j=1}^{\infty} \varphi\left(\mathscr{C}_{j} 2^{k_{j}}\right) 2^{-2 k_{j}}=+\infty
$$

and (27) is true if we put any numbers $n_{j} \geq k_{j}, j=1,2, \ldots$, instead of $k_{j}$. Denote

$$
\mathscr{H}(j, l)=2\left(k_{j} N+l\right)
$$

and $X^{(l)}=\left\{\chi_{\mathscr{C}(j, l)}^{\left(2^{\mathscr{*}(j, l)}-1\right)}\right\}_{j=1}^{\infty}, 1 \leq l \leq N$. With the help of the matrix $S(\mathscr{C})$ for every $B\left(X^{(l)}\right)$, construct an $\mathrm{ON}$ basis as follows:

$$
g_{i}^{(l)}(x)=\sum_{j=1}^{\infty} \zeta_{i j} \chi_{\mathscr{E}(j, l)}^{\left(2^{\mathscr{2}(j, l)}-1\right)}(x) .
$$

From Equations (21)-(26) we immediately have that $\left\{g_{i}^{(l)}\right\}_{i=1}^{\infty}, 1 \leq l \leq N$, are ON systems. By (25) we easily get that $\left\{g_{i}^{(l)}\right\}_{i=1}^{\infty}, 1 \leq l \leq N$, is closed in $B\left(X^{(l)}\right)$. Hence $\left\{g_{i}^{(l)}\right\}_{i=1}^{\infty}$ is an ON basis in $B\left(X^{(l)}\right), 1 \leq l \leq N$. By (4), (26), (22) and (27) we get that every function $g_{1}^{(l)}(x), 1 \leq l \leq N$, is unbounded and their carriers do not intersect. All the functions from $X^{\prime} \backslash X_{1}$, $\left\{\psi_{k}^{(i)}, 2 \leq i \leq 2^{2 k+1}\right\}_{k=0}^{\infty}$ and $\left\{g_{j}^{(l)}\right\}_{j=2}^{\infty}$ are numbered in some order and denoted by $G=\left\{g_{j}\right\}_{j=1}^{\infty}$. From the construction, it is obvious that $B(G \cup \Psi)^{\perp}=$ $B\left(\left\{g_{1}^{(l)}\right\}_{l=1}^{N}\right)$, hence

$$
\operatorname{dim} B(G \cup \Psi)^{\perp}=N
$$

By (22) and (26)-(29) we easily get that

$$
g_{1}^{(l)} \notin \varphi(L), \quad 1 \leq l \leq N .
$$

We construct the desired system $\Phi^{(N)}$ by the systems $G$ and $\Psi$ repeating word for word the reason we used in constructing uniformly bounded ONS $\Phi^{(\infty)}$ by the systems $\left\{g_{j}\right\}_{j=1}^{\infty}$ and $\Psi=\left\{\psi_{k}^{(1)}\right\}_{k=1}^{\infty}$.

In the case $N=\infty$ we make only the following change in the above proof: the system $X$ is broken into infinitely many not intersecting, infinite subsystems $X_{\infty}^{(l)}=\left\{\chi_{m(j, l)}^{\left(2^{m(j, l)}-1\right)}\right\}_{j=1}^{\infty}, l=1,2, \ldots$, such that

$$
m(j, l) \geq k_{j}, \quad l, j=1,2, \ldots,
$$

where $\left\{k_{j}\right\}_{j=1}^{\infty}$ is a subsequence of positive integers for which (27) is true. The remaining part of the proof is the same as for $N<+\infty$. 


\section{REFERENCES}

1. R. D. Mauldin, ed., The Scottish book, Birkhäuser, 1981.

2. S. Kaczmarz, $O$ zupelności ukladów ortogonalnych, in Archiwum Towarzystwa Naukowego we Lwowie, Dzial III, Tom VIII, Zeszyt 5, 431-436.

3. Ulf Grenander and Gabor Szegö, Teoplitz forms and their applications, Univ. of California Press, Los Angeles, CA, 1958.

4. A. M. Olevskii, On an orthonormal system and its applications, Mat. Sb. 71(1966), 297-336; English transl., Transl. Amer. Math. Soc. 76 (1968).

Institute of Mathematics, the Academy of Sciences of the ARmenian SSR, Marshal Bagramian Ave. 24-B, 375019-Yerevan, ARMENIAN SSR, USSR 